\title{
Associations of maternal BMI and insulin resistance with the maternal metabolome and newborn outcomes
}

\author{
Victoria Sandler $^{1}$ • Anna C. Reisetter ${ }^{1}$. James R. Bain ${ }^{2,3,4}$ • Michael J. Muehlbauer ${ }^{2,3,4}$. \\ Michael Nodzenski $^{1}$ - Robert D. Stevens ${ }^{2,3,4}$ • Olga Ilkayeva ${ }^{2,3,4}$ - Lynn P. Lowe ${ }^{1}$. \\ Boyd E. Metzger ${ }^{1}$ • Christopher B. Newgard ${ }^{2,3,4}$ • Denise M. Scholtens ${ }^{1}$. \\ William L. Lowe $\mathrm{Jr}^{1}$ • for the HAPO Study Cooperative Research Group
}

Received: 29 August 2016 / Accepted: 16 November 2016/Published online: 16 December 2016

(C) Springer-Verlag Berlin Heidelberg 2016

\begin{abstract}
Aims/hypothesis Maternal obesity increases the risk for largefor-gestational-age birth and excess newborn adiposity, which are associated with adverse long-term metabolic outcomes in offspring, probably due to effects mediated through the intrauterine environment. We aimed to characterise the maternal metabolic milieu associated with maternal BMI and its relationship to newborn birthweight and adiposity.

Methods Fasting and $1 \mathrm{~h}$ serum samples were collected from 400 European-ancestry mothers in the Hyperglycaemia and Adverse Pregnancy Outcome Study who underwent an OGTT at 28 weeks gestation and whose offspring had anthropometric measurements at birth. Metabolomics assays were performed using biochemical analyses of conventional clinical metabolites, targeted MS-based measurement of amino acids and acylcarnitines and non-targeted GC/MS.

Results Per-metabolite analyses demonstrated broad associations with maternal BMI at fasting and $1 \mathrm{~h}$ for lipids, amino acids and their metabolites together with carbohydrates and organic acids. Similar metabolite classes were associated with
\end{abstract}

Denise M. Scholtens and William L. Lowe Jr are joint senior authors.

Electronic supplementary material The online version of this article (doi:10.1007/s00125-016-4182-2) contains peer-reviewed but unedited supplementary material, which is available to authorised users.

William L. Lowe, Jr

wlowe@northwestern.edu

1 Feinberg School of Medicine, Northwestern University, 420 E. Superior Street, Rubloff 12, Chicago, IL 60611, USA

2 Sarah W. Stedman Nutrition and Metabolism Center, Duke University Medical Center, Durham, NC, USA

3 Duke Molecular Physiology Institute, Durham, NC, USA

4 Duke University School of Medicine, Durham, NC, USA insulin resistance with unique associations including branched-chain amino acids. Pathway analyses indicated overlapping and unique associations with maternal BMI and insulin resistance. Network analyses demonstrated collective associations of maternal metabolite subnetworks with maternal BMI and newborn size and adiposity, including communities of acylcarnitines, lipids and related metabolites, and carbohydrates and organic acids. Random forest analyses demonstrated contribution of lipids and lipid-related metabolites to the association of maternal BMI with newborn outcomes.

Conclusions/interpretation Higher maternal BMI and insulin resistance are associated with broad-based changes in maternal metabolites, with lipids and lipid-related metabolites accounting, in part, for the association of maternal BMI with newborn size at birth.

Keywords Fetal growth $\cdot$ Maternal BMI $\cdot$ Maternal insulin resistance $\cdot$ Maternal metabolism $\cdot$ Pregnancy

$\begin{array}{ll}\text { Abbreviations } \\ \text { BCAA } & \text { Branched-chain amino acid } \\ \text { BW } & \text { Birthweight } \\ \text { FDR } & \text { False discovery rate } \\ \text { FPG } & \text { Fasting plasma glucose } \\ \text { GDM } & \text { Gestational diabetes mellitus } \\ \text { HAPO } & \text { Hyperglycaemia and Adverse } \\ & \text { Pregnancy Outcome } \\ \text { QC } & \text { Quality control } \\ \text { RTL } & \text { Retention-time-locked } \\ \text { SSF } & \text { Sum of skinfolds }\end{array}$




\section{Introduction}

More than one-third of women of reproductive age in the United States are obese, which has serious implications for pregnancy and newborn outcomes [1-3]. Maternal obesity is a risk factor for large-for-gestational-age birth, excess newborn adiposity and adverse long-term metabolic outcomes in offspring [4-7]. Although maternal obesity is associated with higher maternal glucose and gestational diabetes mellitus (GDM) risk, its association with newborn size at birth is, in part, independent of maternal glycaemia $[1,4,8,9]$.

Despite this well-established association between maternal obesity and newborn size at birth and a recent study suggesting a causal relationship between these phenotypes [10], mechanisms underlying this association are poorly defined. To address metabolic linkages between maternal BMI and offspring phenotypes, we characterised the maternal metabolic milieu associated with maternal BMI and its relationship to newborn birthweight (BW) and adiposity.

\section{Study design}

\section{Participants}

We studied 400 mother-offspring dyads of European ancestry from the Hyperglycaemia and Adverse Pregnancy Outcome (HAPO) study. Maternal BMI and glucose, and newborn BW and sum of skinfolds (SSF) spanned the range observed in HAPO [11]. Participants were from the Belfast, UK, and Brisbane and Newcastle, Australia, HAPO field centres, sites used for other HAPO 'omics' investigations [12-14], and included only non-smokers and non-drinkers during pregnancy. All HAPO participants gave informed consent, and all investigations were approved by ethics committees at the participating sites.

\section{Data and sample collection}

HAPO methods were described previously [11, 15]. Eligible women underwent a $75 \mathrm{~g}$ OGTT at 24-32 weeks gestation after an overnight fast of at least $8 \mathrm{~h}$. Fasting (FPG) and $1 \mathrm{~h}$ plasma glucose were measured, and fasting and $1 \mathrm{~h}$ serum samples were stored at $-80^{\circ} \mathrm{C}$ until the metabolomics assays. Insulin sensitivity was estimated using a previously described formula with a modified numerator for scaling [16, 17].

Maternal height and weight were measured at the OGTT study visit. Newborn BW and SSF (sum of flank, triceps and subscapular skinfolds) were measured using standard procedures and calibrated equipment within $72 \mathrm{~h}$ of delivery. Gestational age was determined as described [11, 15]. Demographic characteristics, age, parity and self-identified ethnicity were collected via questionnaire. Participants, caregivers and HAPO staff remained blinded to glucose values unless levels exceeded predefined criteria. 'Unblinded' participants were excluded since participants were medically treated as necessary.

\section{Conventional metabolite and targeted and non-targeted metabolomics assays}

Conventional metabolites were measured as described [13, 17]. Targeted assays of amino acids and acylcarnitines were performed using stable-isotope-labelled internal standards on an Acquity TQD Triple Quadrupole system (Waters Corporation, Milford, MA, USA) [17]. In total, 63 conventional and targeted metabolites were analysed.

Non-targeted assays were performed as described [13, 17]. Methanol, the extraction solvent, was spiked with a retentiontime-locked (RTL) internal standard of perdeuterated myristic acid. Extracts were prepared for GC/MS by methoximation and trimethylsilylation $[17,18]$, and run on a 6890 N GC-5975 Inert MS (Agilent Technologies, Santa Clara, CA, USA). Peaks were deconvoluted with AMDIS freeware [19], and parsed against the Fiehn RTL spectral library [18] with additions from our laboratory. Manual curation included identifying co-eluting groups of isomeric metabolites and selecting reliable peaks [17]. Detected peak areas were $\log _{2}$-transformed for analysis. In total, 76 GC/MS metabolites not assayed using targeted approaches were used for data analysis.

Fasting and $1 \mathrm{~h}$ maternal serum sample pairs were batched for GC/MS. Batches of equal size were run over 50 days and balanced by field centre, maternal glucose and BMI, and newborn outcomes. Quality control (QC) pools were constructed using equal volumes from all maternal samples, prepared for analysis as described above, and injected as first, middle and last samples of each GC/MS run. To control technical variability, GC/MS data were normalised using the metabomxtr $\mathrm{R}$ package [20]. Variations in metabolite levels due to batch and run order identified using QC data were subtracted from analytical sample data to control for technical noise as described [13].

\section{Statistical analysis}

Associations between maternal BMI and maternal fasting and $1 \mathrm{~h}$ metabolites were evaluated using per-metabolite linear models with metabolites as outcomes and BMI or estimated insulin sensitivity as main predictors. For GC/MS metabolites undetectable in $>10 \%$ of samples, mixture models were used to jointly model quantified metabolite abundance with detectability of the metabolite in a sample or lack thereof $[13,20]$. Covariate adjustments were investigated with Model 0 including field centre, gestational age, maternal age and mean arterial pressure at OGTT, newborn sex and sample storage time. Model 1 included Model 0 covariates and glucose at fasting or $1 \mathrm{~h}$ corresponding to metabolite measurement. Model 2 
included Model 0 covariates and insulin sensitivity. Similar analyses were conducted to identify associations between maternal insulin sensitivity and maternal fasting and $1 \mathrm{~h}$ metabolites. Model 0 included the same covariates as Model 0 in BMI analyses, while Model 1 additionally included maternal BMI. Beta estimates and, for linear models, partial correlations were examined across the models for sensitivity to covariate adjustments.

Per-metabolite linear models were also used to investigate associations between maternal metabolites as predictors and newborn SSF and BW outcomes, adjusting for field centre, newborn sex, gestational age at delivery, maternal age, BMI and mean arterial pressure at OGTT and sample storage time. GC/MS metabolites undetectable in $>10 \%$ of samples were treated as four categories with one comprised of undetected values and the others determined by tertiles of observed $\log _{2}-$ peak areas. Associations for the full data set were published previously [13]. All per-metabolite analyses were adjusted for multiple comparisons using adaptive Benjamini-Hochberg false discovery rate (FDR) correction applied separately for targeted and non-targeted data [21]. FDR-adjusted $p$ values $<0.05$ were considered statistically significant.

Pathway analyses were conducted using MetaboAnalyst 3.0 (www.ncbi.nlm.nih.gov/pubmed/25897128, accessed March-May 2016) with human Kyoto Encyclopedia of Genes and Genomes (KEGG) pathways [22] and quantitative enrichment 'globaltest' methodology [23].

Network analyses were conducted to simultaneously model metabolite correlations and phenotype associations. As previously described [13], we constructed partial correlation networks for metabolites at fasting and $1 \mathrm{~h}$ and identified subnetworks jointly associated with our phenotypes. The subnetwork optimisation algorithm we applied used the BioNet R package [24] and applied node scores based on $p$ values for association with individual phenotypes and aggregate $p$ values $\left(p_{a}\right)$ for associations with multiple phenotypes [25]. We first identified subnetworks of maternal fasting and $1 \mathrm{~h}$ metabolites associated with maternal BMI or insulin sensitivity, and then identified subnetworks jointly associated with maternal BMI and newborn outcomes. To summarise local connectivity within subnetworks, spinglass communities, i.e. groups of nodes more densely connected internally than with the rest of the subnetwork, were identified using the igraph $\mathrm{R}$ package [26, 27].

Random forest analyses, data-driven methods designed for prediction [28], were conducted to identify maternal metabolites that improved prediction of newborn BW and SSF beyond maternal BMI and glucose. All random forest analyses were conducted using the party R package [29]. Overall prediction accuracy is measured as 'per cent variation explained'. Contributions of individual predictors are measured by 'variable importance' using a conditional permutation scheme for correlated predictors. Variable importance greater (less) than 0 suggests an increase (decrease) in prediction accuracy. We examined multiple random forest models: $\mathrm{M} 0=$ maternal glucose, gestational age at delivery, field centre, sample storage time; $\mathrm{M} 1=\mathrm{M} 0+$ maternal $\mathrm{BMI} ; \mathrm{M} 2=\mathrm{M} 1+$ highest scoring metabolite within $\mathrm{N}$ spinglass communities for the subnetwork; $\mathrm{M} 3=\mathrm{M} 1+\mathrm{N}$ metabolites with lowest $p_{a} ; \mathrm{M} 4=\mathrm{M} 1+$ metabolites with variable importance $>0$ after running a model including all metabolites with $p_{a}<0.10$. All statistical analyses were conducted using R 3.2.2.

\section{Results}

\section{Study population}

Study population characteristics are shown in ESM Table 1. Mothers spanned the range of maternal BMI and glucose observed in HAPO. Males and females were roughly equally represented among offspring.

\section{Maternal BMI}

Initial analyses examined association of maternal BMI with fasting or $1 \mathrm{~h}$ maternal metabolites using Models 0,1 and 2 described above.

Lipids and lipid metabolites In FDR-adjusted per-metabolite analyses, several lipid metabolites were associated with maternal BMI (Table 1, ESM Fig. 1, ESM Tables 2, 3). The ketone body 3-hydroxybutyrate and its carnitine ester (C4$\mathrm{OH})$ were positively associated with maternal BMI at fasting and $1 \mathrm{~h}$, as were triacylglycerols and glycerol, although the associations of triacylglycerols and $1 \mathrm{~h}$ glycerol were attenuated after adjusting for insulin sensitivity. More abundant fatty acid species such as palmitoleate (C16:1) and oleate (C18:1) were positively associated with maternal BMI at fasting and $1 \mathrm{~h}$, as was $\gamma$-tocopherol at $1 \mathrm{~h}$. Several less abundant fatty acids were negatively associated with maternal BMI at fasting, including saturated fatty acids decanoate $(\mathrm{C} 10: 0)$ and laurate (C12:0) as well as methyl linoleate, a polyunsaturated $n-6$ fatty acid. The $n-3$ fatty acid, docosahexaenoate (C22:6), was negatively associated with maternal BMI both fasting and $1 \mathrm{~h}$ post-glucose. Numerous acylcarnitines were positively associated with maternal BMI at fasting and/or $1 \mathrm{~h}$, including the carnitine esters of palmitoleate and oleate, as well as expected oxidation by-products of these monounsaturated species (C10:1 to C14:1). The carnitine esters of additional polyunsaturated fatty acids were also positively associated with maternal BMI.

Amino acids and metabolites Multiple amino acids were associated with maternal BMI after FDR adjustment (Table 1, ESM Fig. 1, ESM Tables 2, 3). Arginine, asparagine/aspartate, glutamine/glutamate and phenylalanine were 
Table 1 Associations between maternal metabolites and maternal BMI

Metabolite

Maternal fasting metabolites

$\overline{\text { Model } 0} \frac{\text { Model 1 }}{\beta p} \frac{\text { Model 2 }}{\beta p} \frac{\text { Model 0 }}{\beta p} \frac{\text { Model 1 }}{\beta p} \frac{\text { Model 2 }}{\beta p}$

Conventional metabolites

3-Hydroxybutyrate

Glycerol

NEFA

Triacylglycerols

Amino acids (targeted assay)

Arginine

Asparagine/aspartic acid

Glutamine/glutamic acid

Glycine

Leucine/isoleucine

Methionine

Phenylalanine

Valine

Acylcarnitines (targeted assay)

$\mathrm{AC} \mathrm{C} 10$

$\mathrm{AC} \mathrm{C10:1}$

$\mathrm{AC} \mathrm{C10:2}$

$\mathrm{AC}$ C10:3

$\mathrm{AC} \mathrm{C} 12: 1$

AC C14:1

$\mathrm{AC}$ C14:2

$\mathrm{AC}$ C16-OH/C14-DC

$\mathrm{AC} \mathrm{C16:1}$

AC C18:1

AC C18:1-OH/C16:1-DC

$\mathrm{AC} \mathrm{C} 2$

$\mathrm{AC} \mathrm{C} 4-\mathrm{OH}$

$\mathrm{AC} \mathrm{C5}$

$\mathrm{AC}$ C8

AC C8:1

Carbohydrates (non-targeted GC/MS)

Deoxyhexose

Disaccharide

Erythronic acid/threonic acid

Glucose and other aldohexoses

Myoinositol

Ribitol and other pentose alcohols
$+3.2 \times 10^{-6 *}+1.0 \times 10^{-6 *}+1.8 \times 10^{-5 *}+1.5 \times 10^{-11 *}+1.9 \times 10^{-10 *}+6.9 \times 10^{-7} *$

$+7.2 \times 10^{-5 *}+0.0001^{*}+0.0037^{*}+0.0049^{*}+0.016^{*}+0.76$

$+0.27+0.092+0.25+0.034 *+0.15+0.59$

$+0.0030^{*}+0.020^{*}+0.27+0.0007 *+0.0033^{*}+0.32$

$+4.2 \times 10^{-5 *}+0.0005^{*}+0.029^{\dagger}+0.0004^{*}+0.0011^{*}+0.058$

$+1.8 \times 10^{-5 *}+0.0004^{*}+0.021^{\dagger}+0.0001^{*}+0.0006^{*}+0.041^{\dagger}$

$+2.6 \times 10^{-10 *}+3.1 \times 10^{-8 *}+2.8 \times 10^{-6 *}+5.8 \times 10^{-11} *+6.6 \times 10^{-10} *+1.6 \times 10^{-6 *}$

$-0.021^{\dagger}-0.016^{*}-0.077-0.023^{*}-0.025^{\dagger}-0.13$

$+0.38+0.45+0.74+0.029 *+0.060+0.34$

$-0.0007 *-0.0004 *-0.0003 *-0.11 \quad-0.074 \quad-0.060$

$+0.0002 *+0.0020^{*}+0.072+5.8 \times 10^{-5 *}+0.0002 *+0.018^{\dagger}$

$+0.19+0.32+0.87+0.032 *+0.033^{\dagger}+0.31$

$\begin{array}{llll}-0.50 & -0.76+0.52+0.019 *+0.060+0.091\end{array}$

$+0.53+0.50+0.78+0.0024^{*}+0.0079 *+0.025^{\dagger}$

$+0.020^{\dagger}+0.023^{*}+0.18+0.0015^{*}+0.0020^{*}+0.0074 *$

$+0.0004^{*}+0.0009^{*}+0.0014 *+0.0031^{*}+0.0030^{*}+0.0040^{*}$

$+0.018^{\dagger}+0.0043 *+0.0092 *+6.1 \times 10^{-5 *}+0.0003 *+0.0005 *$

$+0.061+0.013^{*}+0.029^{\dagger}+0.0003^{*}+0.0021^{*}+0.0009 *$

$+0.23+0.12+0.26+0.0010 *+0.0040 *+0.0017 *$

$+0.0021 *+0.0024 *+0.0084 * 0.61 * 0.80+0.97$

$+0.0017 *+0.0002 *+0.0004 *+1.1 \times 10^{-7} *+1.3 \times 10^{-6 *}+4.3 \times 10^{-7} *$

$+2.0 \times 10^{-5 *}+7.6 \times 10^{-6 *}+2.4 \times 10^{-5 *}+2.3 \times 10^{-6 *}+1.5 \times 10^{-5 *}+2.2 \times 10^{-5 *}$

$+0.056+0.024^{*}+0.044^{\dagger}+0.0027^{*}+0.0072^{*}+0.0018^{*}$

$+0.058+0.027^{*}+0.031^{\dagger}+0.0035^{*}+0.010^{*}+0.018^{\dagger}$

$+9.9 \times 10^{-6 *}+3.6 \times 10^{-5 *}+6.3 \times 10^{-5 *}+4.0 \times 10^{-6 *}+1.6 \times 10^{-5 *}+0.0002 *$

$+0.027^{\dagger}+0.038^{\dagger}+0.51+0.0012^{*}+0.0016^{*}+0.014^{\dagger}$

$-0.92+0.72+0.96+0.0070^{*}+0.026^{\dagger}+0.048^{\dagger}$

$+1.3 \times 10^{-10 *}+9.4 \times 10^{-10 *}+2.3 \times 10^{-8 *}+7.7 \times 10^{-12 *}+4.1 \times 10^{-11 *}+1.9 \times 10^{-9 *}$

$\begin{array}{lllllll}-0.0050 * & -0.011 * & -0.0062 * & -0.43 & -0.41 & -0.31\end{array}$

$-3.9 \times 10^{-7 *}-6.0 \times 10^{-8 *}-1.7 \times 10^{-8 *}-1.4 \times 10^{-5 *}-8.7 \times 10^{-6 *}-4.3 \times 10^{-7} *$

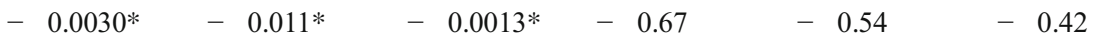

$+0.0042^{*}+0.057+0.24+0.025^{\dagger}+0.33+0.92$

$-2.4 \times 10^{-6 *}-2.3 \times 10^{-5 *}-3.0 \times 10^{-6 *}-0.068-0.052-0.026^{\dagger}$

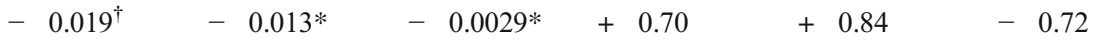

Glycolysis/tricarboxylic acids (non-targeted GC/MS)

Glyceric acid

$-6.0 \times 10^{-5 *}-0.0004 *$

$-0.0004 *-0.012^{\dagger}$

$-0.014^{\dagger}$

$-0.036^{\dagger}$

Fatty acids (non-targeted GC/MS)

Decanoic acid

Docosahexaenoic acid

Lauric acid

$-9.4 \times 10^{-7 *}-9.5 \times 10^{-6 *}$

$-1.6 \times 10^{-6 *}-0.15$

$-0.11$

$-0.042^{\dagger}$

$-8.6 \times 10^{-5 *}-0.0003 *$

$-0.0002 *$

- $0.0021^{*}$

- $0.0025^{*}$

- $0.0026^{*}$

$-0.0003 *-0.0049 *$

- $0.0019^{*}$

$+0.75$

$+0.79$

$-0.72$

- $0.0029^{*}$

- $0.0081^{*}$

$-0.0050 *$

$-0.019^{\dagger}$

$-0.024^{\dagger}$

- 0.067 
Table 1 (continued)

\begin{tabular}{|c|c|c|c|c|c|c|c|c|c|c|c|c|}
\hline \multirow[t]{3}{*}{ Metabolite } & \multicolumn{6}{|c|}{ Maternal fasting metabolites } & \multicolumn{6}{|c|}{ Maternal $1 \mathrm{~h}$ metabolites } \\
\hline & \multicolumn{2}{|c|}{ Model 0} & \multicolumn{2}{|c|}{ Model 1} & \multicolumn{2}{|c|}{ Model 2} & \multicolumn{2}{|c|}{ Model 0} & \multicolumn{2}{|c|}{ Model 1} & \multicolumn{2}{|c|}{ Model 2} \\
\hline & $\beta$ & $p$ & $\beta$ & $p$ & $\beta$ & $p$ & $\beta$ & $p$ & $\beta$ & $p$ & $\beta$ & $p$ \\
\hline Oleic acid & & $0.045^{\dagger}$ & + & $0.011 *$ & & $0.022^{\dagger}$ & + & $0.0002 *$ & + & $0.0016^{*}$ & + & $0.014^{\dagger}$ \\
\hline Palmitoleic acid & + & $0.027^{\dagger}$ & + & $0.0013^{*}$ & + & $0.0018 *$ & + & $0.0004 *$ & + & $0.0022 *$ & + & $0.0007 *$ \\
\hline \multicolumn{13}{|l|}{ Lipids (non-targeted GC/MS) } \\
\hline$\alpha$-Tocopherol & & $0.017^{\dagger}$ & - & $0.014^{*}$ & - & $0.041^{\dagger}$ & - & $0.015^{\dagger}$ & - & $0.016^{\dagger}$ & - & 0.075 \\
\hline$\gamma$-Tocopherol & + & 0.088 & + & 0.11 & + & 0.11 & + & $0.0006^{*}$ & + & $0.0011^{*}$ & + & $0.0008^{*}$ \\
\hline Flavonoid or short-chain cholesteryl ester & + & $0.020^{\dagger}$ & + & $0.0074 *$ & + & $0.018^{\dagger}$ & + & 0.15 & + & 0.17 & + & 0.059 \\
\hline \multicolumn{13}{|l|}{ Amino acids (non-targeted GC/MS) } \\
\hline 3-Indoleacetic acid & - & $0.0091^{*}$ & - & $0.018^{*}$ & - & $0.0027^{*}$ & - & 0.42 & - & 0.47 & - & 0.14 \\
\hline 3-Indolelactic acid/tryptophan & - & $0.0080^{*}$ & - & $0.013 *$ & - & $0.0040 *$ & - & $0.0010^{*}$ & - & $0.0017 *$ & - & $0.0041 *$ \\
\hline Aminomalonic acid & - & $0.017^{\dagger}$ & - & $0.017 *$ & - & $0.031^{\dagger}$ & - & 0.46 & - & 0.38 & - & 0.44 \\
\hline Urea & - & $3.1 \times 10^{-5 *}$ & - & $3.4 \times 10^{-5 *}$ & - & $0.0001 *$ & - & $0.0058^{*}$ & - & $0.011^{\dagger}$ & - & $0.019^{\dagger}$ \\
\hline \multicolumn{13}{|l|}{ Other metabolites (non-targeted GC/MS) } \\
\hline$p$-Cresol & - & $0.0057^{*}$ & - & $0.0022 *$ & - & $0.0006^{*}$ & - & $0.0065 *$ & - & $0.014^{\dagger}$ & - & $0.032^{\dagger}$ \\
\hline Phosphoric acid & + & $0.013^{*}$ & + & $0.0094 *$ & + & $0.016^{\dagger}$ & + & $0.0009 *$ & + & $0.0017 *$ & + & $0.0006^{*}$ \\
\hline
\end{tabular}

*FDR-adjusted $p<0.05$

${ }^{\dagger}$ Nominal $p<0.05$

Model 0 adjusted for field centre, gestational age, maternal age and mean arterial pressure at OGTT, newborn sex and sample storage time. Model 1 included Model 0 covariates and fasting glucose. Model 2 included Model 0 covariates and insulin sensitivity

positively associated with maternal BMI at fasting and $1 \mathrm{~h}$ in Models 0 and 1 . Only glutamine/glutamate was positively associated with BMI in Model 2. Glycine demonstrated a significant or nominally significant negative association with maternal BMI at fasting and $1 \mathrm{~h}$ in Models 0 and 1 but not Model 2. Fasting but not $1 \mathrm{~h}$ methionine was negatively associated with maternal BMI in all three models. The branchedchain amino acids (BCAAs) leucine/isoleucine and valine were positively associated with maternal BMI at $1 \mathrm{~h}$ but their association was attenuated after adjusting for glucose or insulin sensitivity. Isovalerylcarnitine (C5), a product of leucine metabolism, was positively associated with maternal BMI in Models 0 and 1 at $1 \mathrm{~h}$. Fasting $p$-cresol, an end-product of protein breakdown and gut microbial metabolism, and urea, a product of amino acid metabolism, were negatively associated with maternal BMI in all models.

Sugars, organic acids and phosphate Multiple sugars and organic acids were associated with maternal BMI after FDR adjustment (Table 1, ESM Fig. 1, ESM Tables 2, 3). Disaccharides were negatively associated with maternal BMI at fasting and $1 \mathrm{~h}$; glyceric acid was negatively associated only at fasting. Phosphoric acid was positively associated with maternal BMI at both time points in all three models. Fasting deoxyhexose, erythronic acid/threonic acid, ribitol and myoinositol were all negatively associated with maternal BMI at fasting but not $1 \mathrm{~h}$. Original HAPO glucose measurements, as well as the GC/MS peak for glucose and other aldohexoses in Model 0, confirm a positive association with maternal BMI at fasting and $1 \mathrm{~h}$ (data not shown for original HAPO measurements).

\section{Maternal insulin sensitivity}

Similar FDR-adjusted per-metabolite associations were examined with estimated insulin sensitivity. At fasting, BCAAs and their metabolites, isovalerylcarnitine (C5) and 3hydroxyisovalerylcarnitine (C5-OH/C3-DC), were associated with greater insulin resistance (Table 2, ESM Fig. 2, ESM Tables 4, 5). Alanine, arginine, asparagine/aspartate, glutamine/glutamate, phenylalanine and proline as well as triacylglycerols, lactate and dihydroxybutanoate, a metabolite of the short-chain fatty acid butyrate, were also associated with greater insulin resistance before and after adjusting for BMI. In contrast, glycerol, octenoyl carnitine (C8:1) and decadienoyl carnitine (C10:2) were associated with maternal insulin resistance in Model 0 but not Model 1. The only metabolites positively associated with maternal insulin sensitivity were a long- $(\mathrm{C} 14: 1-\mathrm{OH})$ and medium-chain $(\mathrm{C} 8: 1-\mathrm{OH} / \mathrm{C} 6: 1-$ DC) carnitine ester.

Similar associations were evident at $1 \mathrm{~h}$, except for C14:1OH, C8:1-OH/C6:1-DC and C10:2 (Table 2, ESM Fig. 2, ESM Tables 4, 5). In addition, $1 \mathrm{~h}$ NEFA, oleate, 3-hydroxybutyrate and its carnitine ester $\mathrm{C} 4-\mathrm{OH}$ (in Model 0) were associated with greater maternal insulin resistance. Furthermore, $1 \mathrm{~h}$ 
Table 2 Associations between maternal metabolites and maternal insulin sensitivity

\begin{tabular}{|c|c|c|c|c|c|c|c|c|}
\hline \multirow[t]{3}{*}{ Metabolite } & \multicolumn{4}{|c|}{ Maternal fasting metabolites } & \multicolumn{4}{|c|}{ Maternal $1 \mathrm{~h}$ metabolites } \\
\hline & \multicolumn{2}{|c|}{ Model 0} & \multicolumn{2}{|c|}{ Model 1} & \multicolumn{2}{|c|}{ Model 0} & \multicolumn{2}{|c|}{ Model 1} \\
\hline & $\beta$ & $p$ & $\beta$ & $p$ & $\beta$ & $p$ & $\beta$ & $p$ \\
\hline \multicolumn{9}{|l|}{ Conventional metabolites } \\
\hline 3-Hydroxybutyrate & - & 0.056 & + & 0.99 & - & $1.0 \times 10^{-8 *}$ & - & $0.0007 *$ \\
\hline Glycerol & - & $0.0003^{*}$ & - & 0.054 & - & $1.8 \times 10^{-11 *}$ & - & $4.0 \times 10^{-9} *$ \\
\hline Lactate & - & $0.0001 *$ & - & $0.0002 *$ & - & $2.1 \times 10^{-9} *$ & - & $1.9 \times 10^{-8 *}$ \\
\hline NEFA & - & 0.80 & + & 0.75 & - & $5.8 \times 10^{-5 *}$ & - & $0.0004^{*}$ \\
\hline Triacylglycerols & - & $4.0 \times 10^{-7} *$ & - & $3.2 \times 10^{-5 *}$ & - & $2.2 \times 10^{-10_{*}}$ & - & $6.6 \times 10^{-8 *}$ \\
\hline \multicolumn{9}{|l|}{ Amino acids (targeted assay) } \\
\hline Alanine & - & $0.0007^{*}$ & - & $0.0002 *$ & - & $0.0007 *$ & - & $0.0017 *$ \\
\hline Arginine & - & $9.4 \times 10^{-9} *$ & - & $6.6 \times 10^{-6 *}$ & - & $3.6 \times 10^{-7 *}$ & - & $7.1 \times 10^{-5 *}$ \\
\hline Asparagine/aspartic acid & - & $8.4 \times 10^{-9} *$ & - & $6.8 \times 10^{-6 *}$ & - & $1.5 \times 10^{-7} *$ & - & $4.1 \times 10^{-5 *}$ \\
\hline Glutamine/glutamic acid & - & $3.9 \times 10^{-8} *$ & - & $0.0006^{*}$ & - & $7.0 \times 10^{-9} *$ & - & $0.0003 *$ \\
\hline Glycine & + & 0.092 & + & 0.41 & + & $0.029 *$ & + & 0.16 \\
\hline Leucine/isoleucine & - & $0.0025^{*}$ & - & $0.0043 *$ & - & $0.0010 *$ & - & $0.0083 *$ \\
\hline Phenylalanine & - & $2.0 \times 10^{-9} *$ & - & $1.8 \times 10^{-6} *$ & - & $1.0 \times 10^{-7} *$ & - & $7.6 \times 10^{-5} *$ \\
\hline Proline & - & $2.1 \times 10^{-6 *}$ & - & $6.0 \times 10^{-6 *}$ & - & $1.5 \times 10^{-7} *$ & - & $1.4 \times 10^{-6 *}$ \\
\hline Valine & - & $0.0019^{*}$ & - & $0.0082 *$ & - & $0.0010 *$ & - & $0.014^{\dagger}$ \\
\hline \multicolumn{9}{|l|}{ Acylcarnitines (targeted assay) } \\
\hline $\mathrm{AC}$ C10:1 & - & 0.30 & - & 0.48 & - & $0.0079^{\mathrm{a}}$ & - & 0.15 \\
\hline AC C10:2 & - & $0.0030^{*}$ & - & $0.036^{\dagger}$ & - & 0.075 & - & 0.57 \\
\hline AC C14:1-OH & + & $0.0075^{*}$ & + & $0.0028 *$ & + & 0.16 & + & 0.084 \\
\hline $\mathrm{AC} \mathrm{C} 4-\mathrm{OH}$ & - & 0.058 & - & 0.88 & - & $0.0020 *$ & - & 0.19 \\
\hline $\mathrm{AC} \mathrm{C} 4 / \mathrm{Ci} 4$ & - & 0.17 & - & 0.51 & - & $0.028^{*}$ & - & 0.16 \\
\hline $\mathrm{AC} \mathrm{C5}$ & - & $2.6 \times 10^{-5} *$ & - & $0.0005 *$ & - & $0.0091 *$ & - & 0.21 \\
\hline $\mathrm{AC}$ C5-DC & - & 0.070 & - & 0.11 & - & $0.014 *$ & - & $0.028^{\dagger}$ \\
\hline $\mathrm{AC} \mathrm{C} 5-\mathrm{OH} / \mathrm{C} 3-\mathrm{DC}$ & - & $0.0001^{*}$ & - & $0.0005 *$ & - & $0.0037^{*}$ & - & $0.017^{\dagger}$ \\
\hline $\mathrm{AC} \mathrm{C} 8$ & + & 0.69 & + & 0.70 & - & $0.026^{*}$ & - & 0.21 \\
\hline AC C8:1 & - & $0.0008^{*}$ & - & 0.39 & - & $0.0009 *$ & - & 0.44 \\
\hline AC C8:1-OH/C6:1-DC & + & $0.017 *$ & - & 0.076 & + & 0.062 & + & 0.18 \\
\hline \multicolumn{9}{|l|}{ Carbohydrates (non-targeted GC/MS) } \\
\hline Dihydroxybutanoic acid & - & $0.0009^{*}$ & - & $6.2 \times 10^{-5 *}$ & - & $0.0002 *$ & - & $0.0002 *$ \\
\hline Fructose or similar ketohexose & - & $0.0050^{\dagger}$ & - & $0.0018 *$ & - & $1.6 \times 10^{-5 *}$ & - & $2.3 \times 10^{-5} *$ \\
\hline Gluconate & - & 0.33 & - & 0.13 & - & $1.1 \times 10^{-14} *$ & - & $1.1 \times 10^{-13 *}$ \\
\hline Glucose and other aldohexoses & - & $3.8 \times 10^{-6} *$ & - & $0.0002 *$ & - & $6.0 \times 10^{-9} *$ & - & $1.5 \times 10^{-7} *$ \\
\hline Hexitols & - & 0.050 & - & 0.078 & - & $0.0002 *$ & - & $0.0002 *$ \\
\hline \multicolumn{9}{|l|}{ Fatty acids (non-targeted GC/MS) } \\
\hline Oleic acid & + & 0.91 & + & 0.28 & - & $0.0003 *$ & - & $0.017^{\dagger}$ \\
\hline \multicolumn{9}{|l|}{ Lipids (non-targeted GC/MS) } \\
\hline Campesterol & + & 0.071 & + & 0.17 & + & $0.0050^{*}$ & + & $0.034^{\dagger}$ \\
\hline \multicolumn{9}{|l|}{ Other metabolites (non-targeted GC/MS) } \\
\hline$O$-Methylphosphate & - & 0.42 & - & 0.24 & - & $0.0022 *$ & - & $0.0032 *$ \\
\hline
\end{tabular}

*FDR-adjusted $p<0.05$

${ }^{\dagger}$ Nominal $p<0.05$

Model 0 adjusted for field centre, gestational age, maternal age and mean arterial pressure at OGTT, newborn sex and sample storage time. Model 1 included Model 0 covariates and maternal BMI 
campesterol, a phytosterol, was associated with greater insulin sensitivity. Fasting and $1 \mathrm{~h}$ dihydroxybutanoate and fructose were associated with maternal insulin resistance, as were $1 \mathrm{~h}$ gluconate, hexitols and $O$-methylphosphate.

\section{Pathway analyses}

Pathway analyses at fasting and $1 \mathrm{~h}$ identified multiple amino acid, lipid, and sugar and carbohydrate metabolism pathways with metabolite members jointly associated with maternal BMI and/or insulin sensitivity (ESM Tables 6, 7). Pathways associated with maternal BMI and insulin sensitivity overlapped in several instances; however, exceptions included pathways for BCAA degradation and glycolysis or gluconeogenesis for insulin resistance only and tryptophan metabolism and fatty acid biosynthesis for BMI only.

\section{Maternal BMI and insulin sensitivity networks}

To complement per-metabolite analyses, network analyses were performed to identify correlated metabolites demonstrating joint association with phenotype(s). The subnetwork of fasting metabolites associated with maternal BMI included six spinglass communities (i.e. groups of metabolites within subnetworks more densely connected internally than with the remaining subnetwork) composed largely of amino acids, medium-chain acylcarnitines, long-chain acylcarnitines and lipid metabolites, or mixed metabolites including carbohydrates, organic acids and other metabolites (Fig. 1). Spinglass communities for $1 \mathrm{~h}$ metabolites were similar, composed largely of amino acids and their metabolites, acylcarnitines and lipid-related metabolites, or clusters of mixed metabolites.

Spinglass communities in the maternal insulin sensitivity network differed from the BMI network (Fig. 2). A few medium- and long-chain acylcarnitines and palmitoleate were positively associated with insulin sensitivity at fasting, while remaining metabolites were negatively associated. Similar to non-pregnant populations [30,31], interconnected communities of BCAAs and acylcarnitines generated during BCAA catabolism were associated with insulin resistance. The aromatic amino acid phenylalanine together with asparagine/aspartate, arginine, glutamine/glutamate and proline were also associated with insulin resistance, as were a community comprised largely of sugars and a small community including triacylglycerols and cholesterol. At $1 \mathrm{~h}$, communities of sugars and organic acids, amino acids including BCAAs, and lipids and related metabolites were the major large communities associated with insulin resistance. A small community including lactate and pyruvate and one including organic acids were also associated with insulin resistance.

\section{Maternal BMI and newborn outcome networks}

Estimated insulin sensitivity in HAPO mothers, in contrast to maternal BMI [1, 4], was not associated with BW or SSF after adjusting for BMI (data not shown). Thus, subsequent analyses focused on networks jointly associated with maternal BMI and newborn outcomes. Networks of fasting metabolites associated with both maternal BMI and either newborn SSF or BW were smaller than networks associated with maternal BMI only but consisted of similar communities of acylcarnitines and lipid metabolites, amino acids plus triacylglycerols and mixed metabolites (Fig. 3). The $1 \mathrm{~h}$ networks were generally smaller than fasting networks and again consisted of communities composed largely of acylcarnitines and fatty acids together with lipids or a mix of metabolites.

\section{Random forest analyses}

Random forest analyses were performed to identify maternal metabolites associated with maternal BMI that may predict newborn BW and/or SSF (Fig. 4, ESM Tables 8-11). M1, which included maternal BMI, explained higher overall per cent variation for both newborn outcomes than M0, which included maternal glucose and other baseline covariates. This reflects an independent association of maternal BMI with newborn size outcomes. The relative contribution of maternal BMI as a predictor of newborn outcomes (measured by conditional variable importance) decreased in M2, M3 and M4 compared with M1 as additional metabolites were included in the models (Fig. 4). This decrease suggests that maternal metabolites correlated with maternal BMI account in part for the association of BMI with these newborn outcomes. In the fasting state, these include a number of lipid-related metabolites (triacylglycerols, a carnitine ester of 3-hydroxybutyrate, long-chain acylcarnitines and glycerol-1-phosphate), amino acids and their metabolites, and organic acids; at $1 \mathrm{~h}$ these include primarily lipids and lipid-related metabolites (triacylglycerols, fatty acids, 3-hydroxybutyrate, and medium- and long-chain acylcarnitines, among others), amino acid metabolites and sugars/alcohols.

\section{Discussion}

We examined associations of maternal BMI and insulin resistance with the maternal metabolome using targeted and nontargeted metabolomics. Metabolomic studies of maternal metabolism during pregnancy have focused largely on normal pregnancy and GDM [32-38], not maternal BMI, although biomarkers that may distinguish obese from normal weight mothers during the first trimester of pregnancy were recently reported [2]. In the present study, per-metabolite, network, pathway and random forest analyses demonstrated broad- 

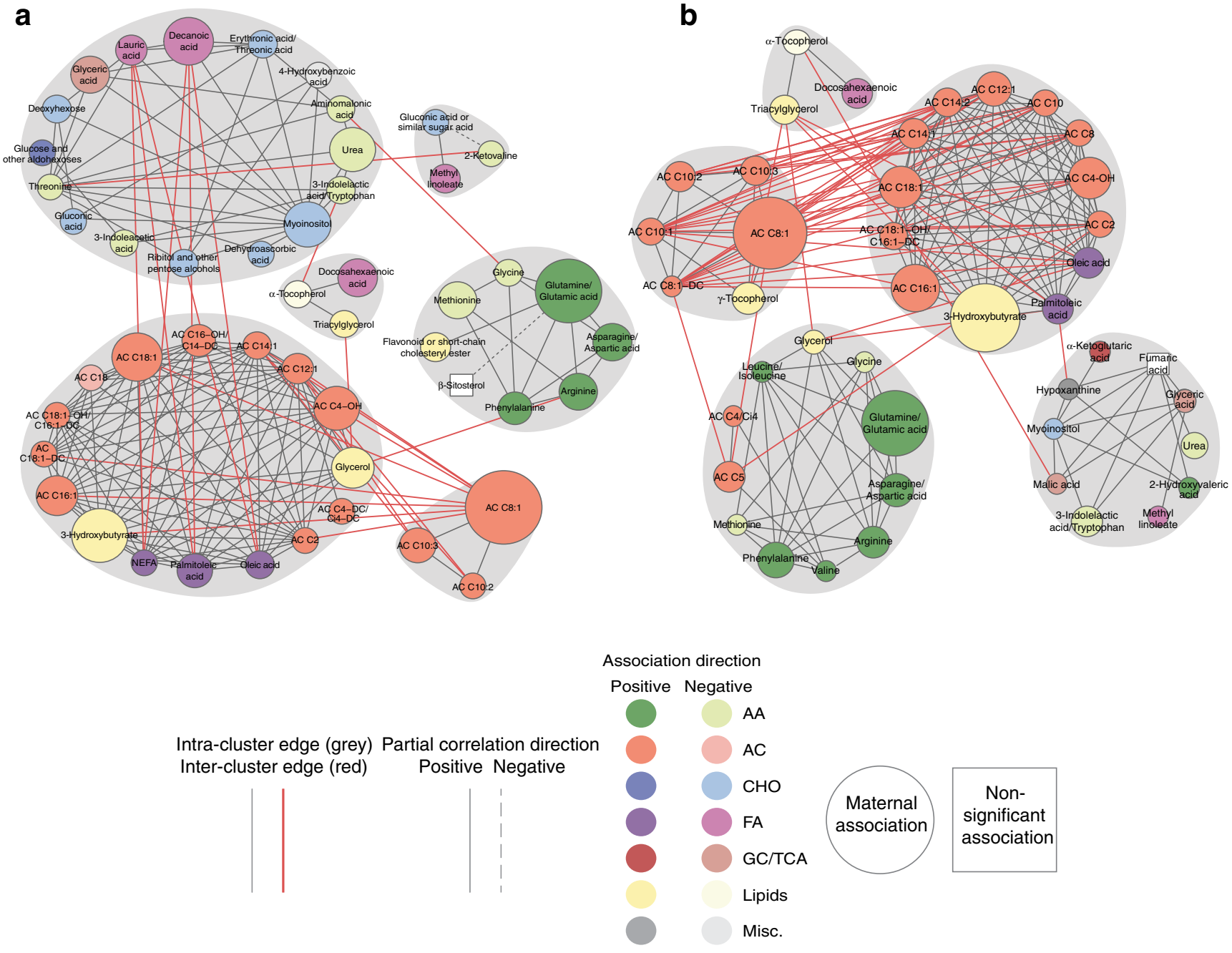

Fig. 1 Subnetworks of maternal (a) fasting and (b) $1 \mathrm{~h}$ metabolites associated with maternal BMI, adjusted for maternal glucose at corresponding time points. Edges represent partial correlations with magnitude $>0.25$. Nodes are sized by strength of association, shaded by direction of association, and coloured by metabolite class (AA, amino acid; AC,

based associations of the maternal metabolome with higher maternal BMI and insulin resistance.

We found similarities and differences from metabolomic studies focused on obesity or BMI in non-pregnant populations [30, 39-42]. Although numerous lipid-related metabolites and amino acids are associated in both populations, one notable difference was the absence of association of fasting tyrosine or BCAA with maternal BMI, while BCAA $1 \mathrm{~h}$ after glucose were associated with BMI only before adjusting for $1 \mathrm{~h}$ glucose or insulin sensitivity. Findings with some lipids and related metabolites also differed. In non-pregnant populations, 3hydroxybutyrate was negatively or not associated with BMI; fatty acids were generally positively associated. In pregnancy, 3-hydroxybutyrate was positively associated with maternal BMI while medium- and long-chain fatty acids demonstrated a mix of negative and positive associations, similar to recently reported negative and positive associations of long-chain fatty

acylcarnitine; CHO, carbohydrate; FA, fatty acid; GC/TCA, glycolysis/ tricarboxylic acid cycle, Misc., miscellaneous). Grey shading denotes spinglass communities. These subnetworks indicate correlated metabolites jointly associated with maternal BMI

acids with maternal BMI at $\sim 20$ weeks gestation [43]. Enhanced placental transfer of select fatty acids important for fetal development, e.g. docosahexanoic acid [44], may contribute to differences with the non-pregnant state.

Association of maternal metabolites with insulin resistance during pregnancy has not been reported. Similar to non-pregnant cohorts [41, 45], we found a negative association of BCAA, alanine, proline and glutamine/glutamate, and no association of NEFA with insulin sensitivity. Different from non-pregnant cohorts, ornithine, tyrosine and glycine (except for $1 \mathrm{~h}$ post-glucose before adjusting for maternal BMI) were not associated with insulin sensitivity, although statistical power may have limited our ability to detect modest effects due to multiple comparisons corrections [41]. We also demonstrated a negative association of arginine, medium-chain acylcarnitines and BCAA metabolites with insulin sensitivity. 

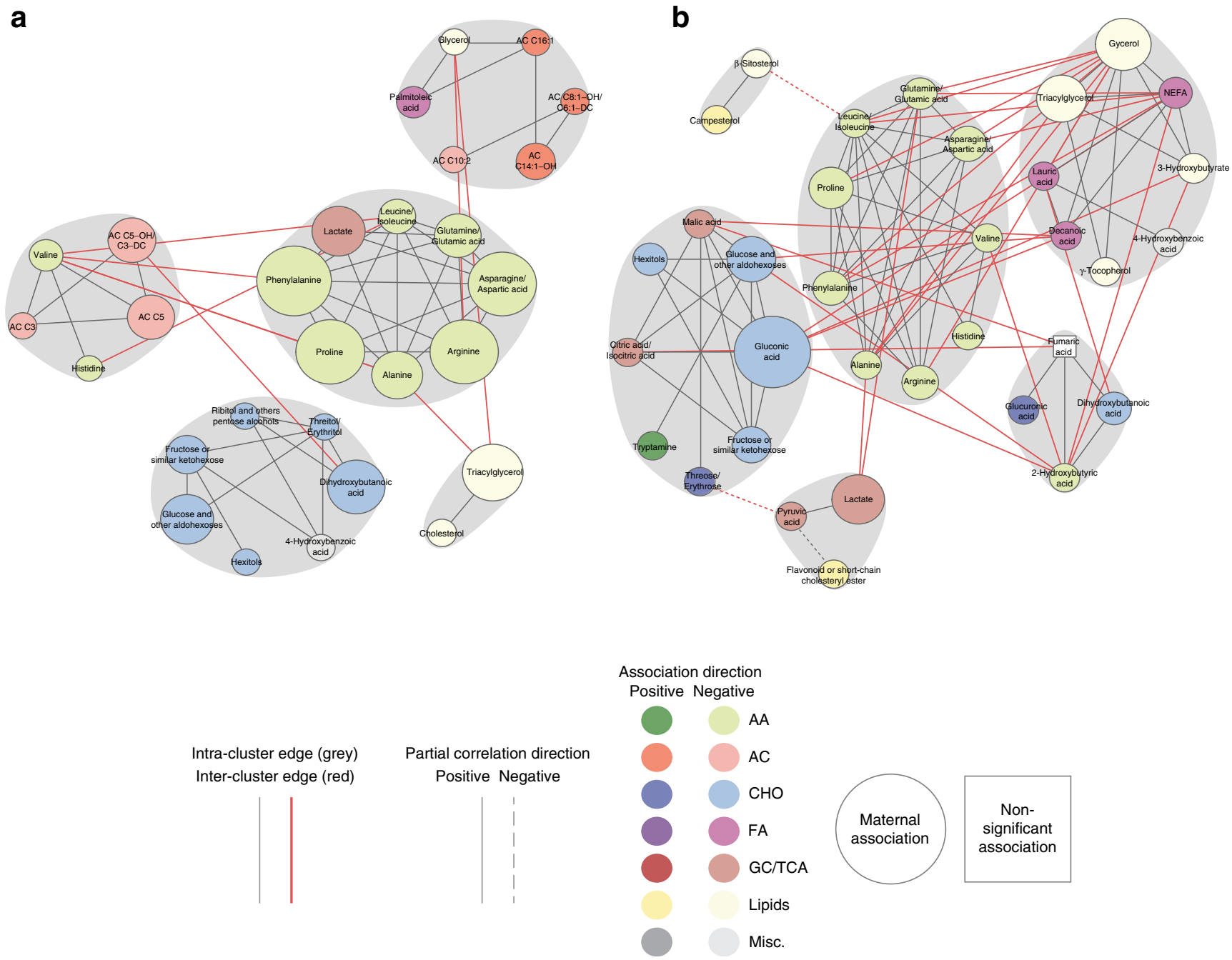

Fig. 2 Subnetworks of maternal (a) fasting and (b) $1 \mathrm{~h}$ metabolites associated with maternal insulin sensitivity adjusted for maternal BMI. Plotting features are as described in Fig. 1. These subnetworks indicate correlated metabolites jointly associated with maternal insulin sensitivity

BMI, glucose and insulin sensitivity are interrelated; pregnancy also markedly decreases insulin sensitivity [46]. To try to define the metabolome independently associated with these phenotypes, we adjusted for the other phenotypes when one was used as a predictor. Adjusting for maternal glucose modestly impacted the maternal metabolome associated with BMI; however, the maternal metabolome associated with BMI or insulin sensitivity was more significantly impacted after adjusting for the other measure. Insulin sensitivity explained the association of triacylglycerols as well as a number of amino acids, including the BCAA, with maternal BMI. Maternal BMI but not insulin sensitivity was associated with a number of fasting fatty acids. Network analyses confirmed these differences with more fatty acids and their carnitine esters associated with BMI compared with insulin sensitivity. Finally, although similar pathways were associated with maternal BMI and insulin sensitivity, fatty acid biosynthesis was uniquely associated with maternal BMI, while BCAA degradation pathways were uniquely associated with maternal insulin sensitivity. The associations between BCAA and insulin sensitivity are of interest given the growing number of studies demonstrating cause and effect relationships between BCAA levels and metabolism and insulin sensitivity [30, 47, 48].

The association of maternal BMI with newborn size at birth is well established $[1,4,10]$, but the lack of association of insulin resistance corrected for maternal BMI with newborn BW and SSF is new. This dichotomy may provide important clues for defining maternal metabolic variables with causal links to newborn outcomes. For example, BCAA and their metabolites were strongly associated with insulin sensitivity in pregnant and non-pregnant individuals, but in contrast to the situation in non-pregnant individuals, BCAA and their metabolites were not associated with BMI in pregnant women, suggesting that they might not be the primary effectors of the fetal outcomes reported here. Instead, multiple markers of fasting lipid metabolism were clearly associated with maternal BMI, including several abundant long-chain fatty acids and acylcarnitines and classical markers of lipolysis and fatty acid 
a
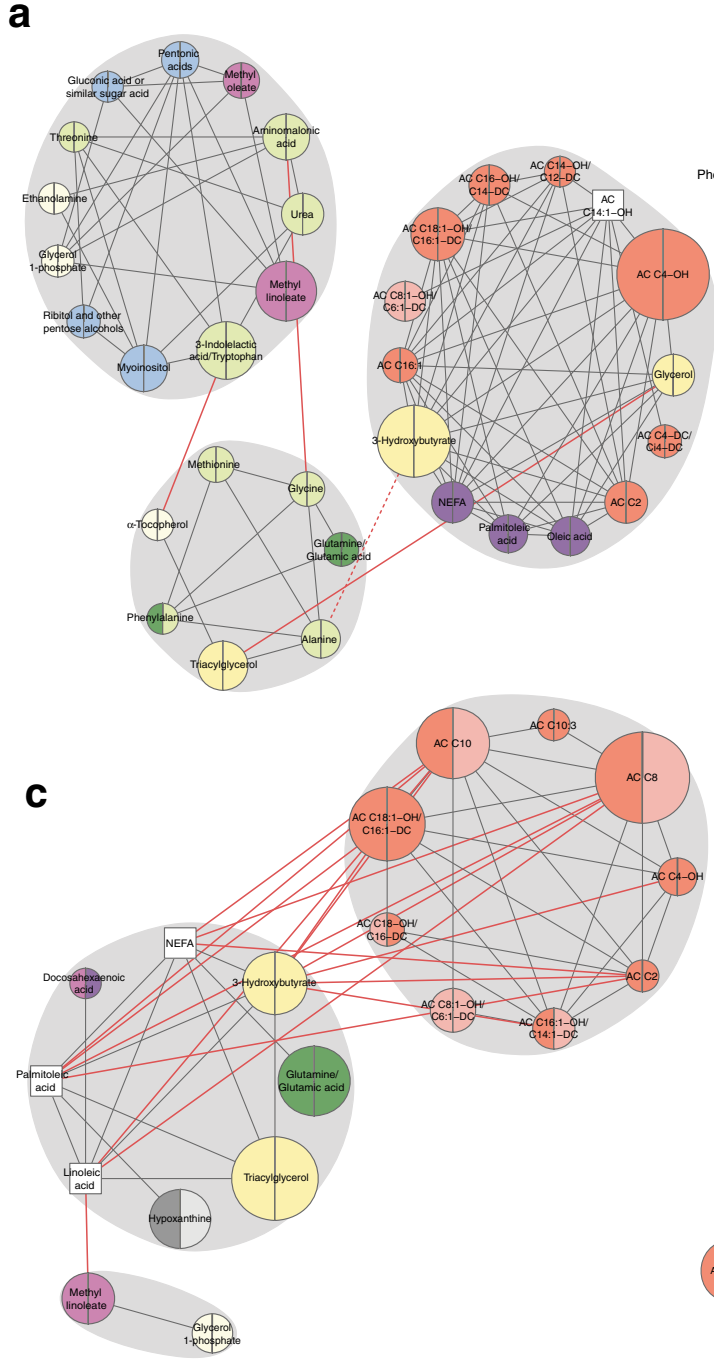

b
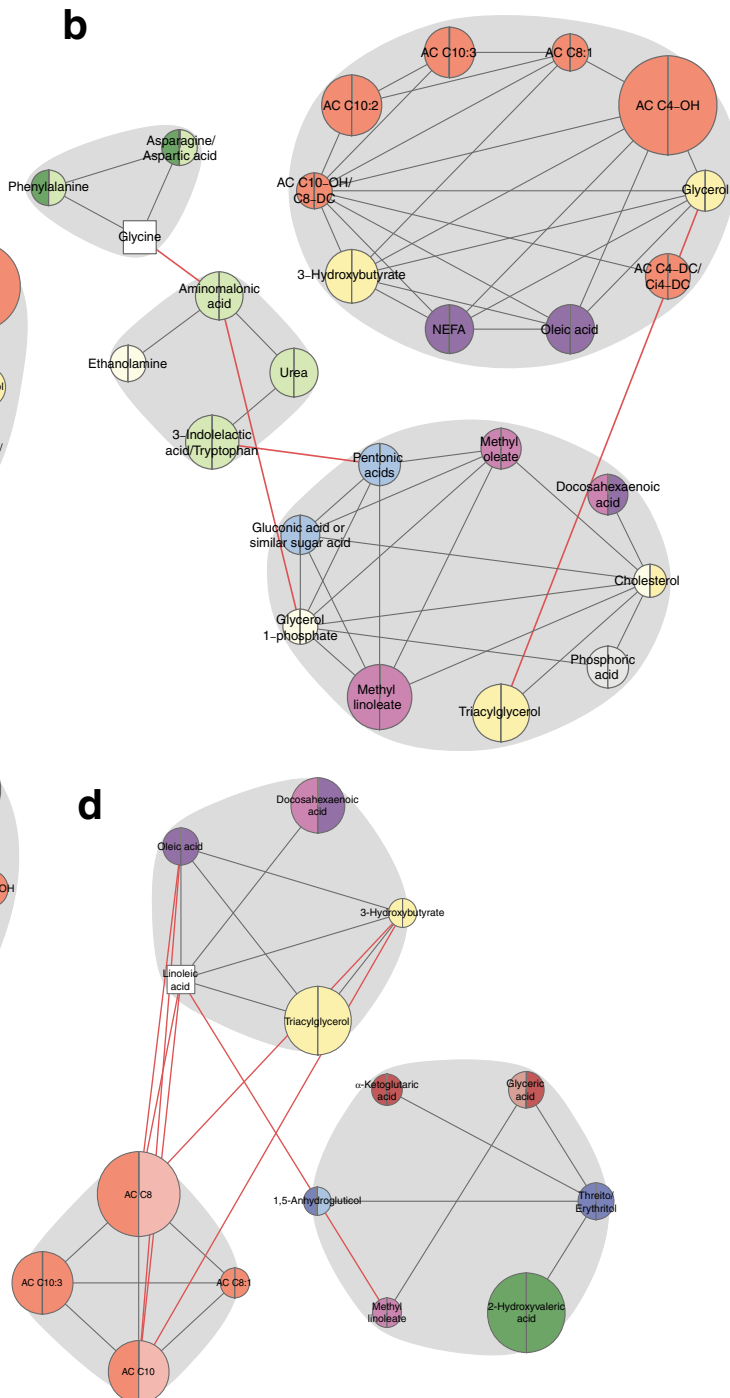

Partial correlation direction Positive Negative

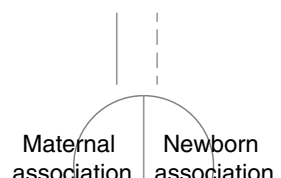
association association

Intra-cluster edge (grey) Inter-cluster edge (red)

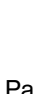
direction e Negative

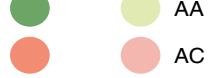

C $\mathrm{CHO}$

- FA

GC/TCA

Lipids

Misc.

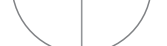

Non-

significant association
Fig. 3 Subnetworks of maternal metabolites associated with both maternal BMI adjusted for maternal glucose and newborn outcomes. Plotting features are as described in Fig. 1. Subnetwork of maternal (a) fasting and (c) $1 \mathrm{~h}$ metabolites associated with both maternal BMI and newborn SSF.

oxidation, e.g. 3-hydroxybutyrate and glycerol. Moreover, network analyses demonstrated that most of these analytes were associated with newborn BW and SSF in both fasting and $1 \mathrm{~h}$ post-glucose samples (Fig. 3), suggesting that increased lipid supply associated with higher BMI results in increased maternal fatty acid oxidation with accumulation of acylcarnitines and ketones as by-products. This greater reliance on fatty acid oxidation for energy needs may reduce catabolism of other fuels, including glucose and amino acids, sparing them for transfer to the fetus. Indeed, network analyses demonstrated negative rather than positive association of several amino acids and related metabolites with newborn outcomes in the context of maternal BMI. Together, these findings suggest that by-products of lipid metabolism generated in high BMI mothers may influence fetal growth and adiposity. This idea will require further investigation;
Subnetwork of maternal (b) fasting and (d) $1 \mathrm{~h}$ metabolites associated with both maternal BMI and BW. These subnetworks indicate correlated metabolites that are jointly associated with both maternal BMI and newborn outcomes

however, we note that ketone bodies are efficiently transported across the placenta and available to the fetus either as oxidative fuels or for fatty acid synthesis [49], while docosahexaenoic acid was negatively associated with maternal BMI but positively associated with both newborn outcomes, consistent with transplacental transport to support brain and overall development in the growing fetus [44].

Plasma glucose and BMI are interrelated but their associations with newborn size at birth are, in part, independent $[1,4,8$, 9]. Consistent with this, network analyses of maternal fasting and $1 \mathrm{~h}$ metabolites jointly associated with maternal BMI and newborn outcomes show differences from our previous network analyses of maternal glycaemia [13]. For example, spinglass communities comprised largely of fasting amino acids were identified when BMI but not FPG was the maternal phenotype. One hour post-glucose, communities comprised largely 

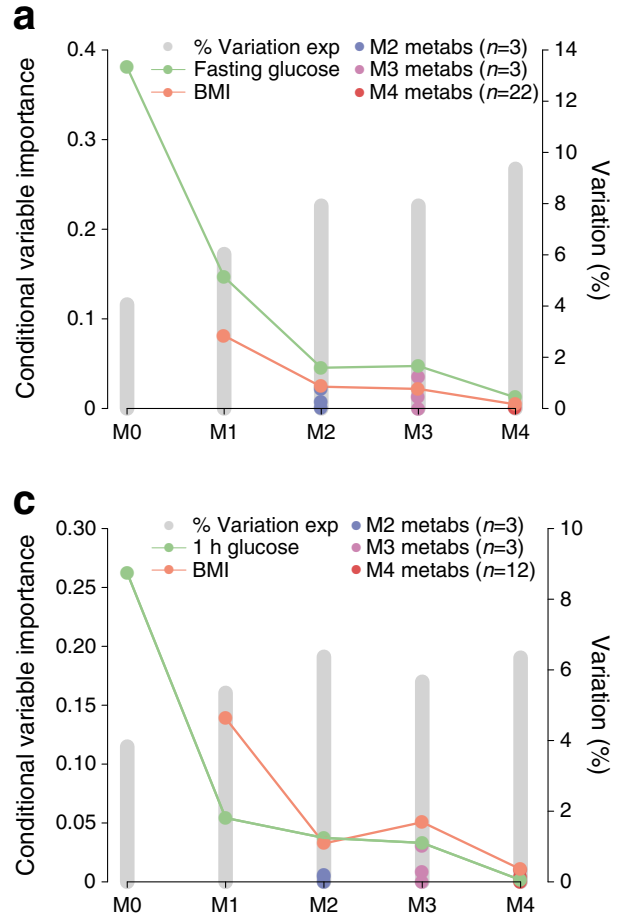

Fig. 4 Plots of conditional variable importance (left-hand $y$-axis) and per cent variation explained (right-hand $y$-axis) for random forest models M0-M4. Green (M0-M4) and orange (M1-M4) points and lines track conditional variable importance for maternal glucose and BMI, respectively. Blue, pink and red points correspond to conditional variable importance of metabolites in M2, M3 and M4, respectively. Grey bars plot

of carbohydrates were present with $1 \mathrm{~h}$ glucose as the maternal phenotype, while communities with amino acid-related metabolites were present when maternal BMI was the phenotype. Fasting and $1 \mathrm{~h}$ post-glucose, spinglass communities comprised of fasting acylcarnitines, fatty acids, lipids and/or lipid-related metabolites were identified for both maternal phenotypes, but there were differences in the metabolites present in the communities. Random forest analysis also identified a broad array of metabolites associated with maternal BMI that predict BW and SSF independent of maternal BMI and glucose (see ESM Tables 8-11). In previous random forest analyses [13], we showed that some of these same metabolites were associated with maternal glucose and predicted BW and/or SSF. However, some metabolites were uniquely associated with maternal BMI and predicted BW and/or SSF, including fasting 3hydroxybutyrate and its carnitine ester, cholesterol, acetylcarnitine and asparagine/aspartate as well as $1 \mathrm{~h}$ docosaheaenoate, 3-hydroxybutyrate, 2-hydroxyvalerate and $\alpha$-ketoglutarate. Together, these data suggest that the associations of BMI and maternal glycaemia with newborn size at birth can be accounted for, in part, by different groups of metabolites, consistent with their partially independent effects on newborn outcomes.

Our study had several strengths. It is the largest to examine the associations of maternal BMI on the maternal metabolome and the first to consider estimated maternal insulin sensitivity b
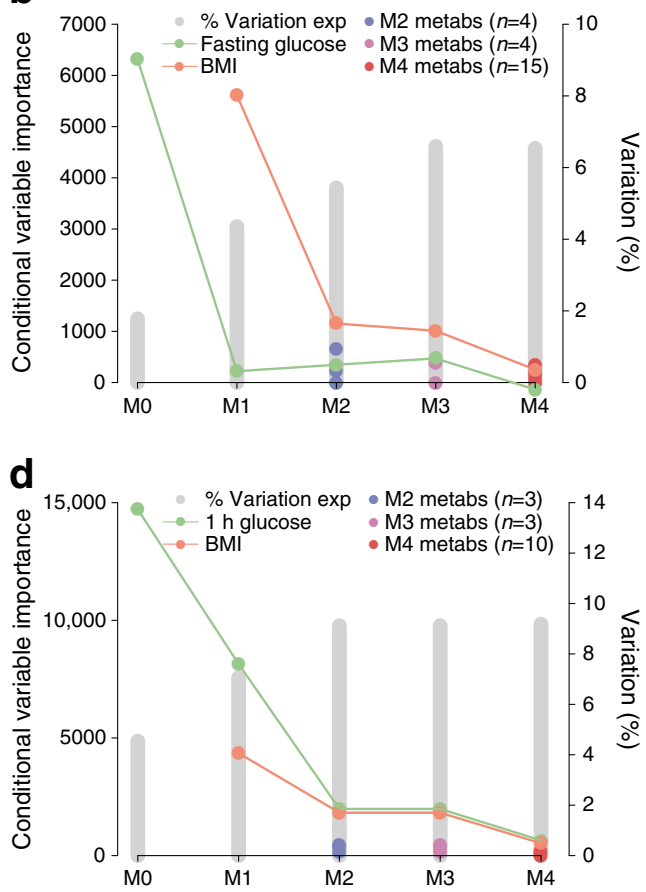

overall per cent variation explained by all predictors in M0-M4. (a) Fasting metabolites and newborn SSF. (b) Fasting metabolites and BW. (c) $1 \mathrm{~h}$ metabolites and newborn SSF. (d) $1 \mathrm{~h}$ metabolites and BW. These plots demonstrate the contribution of fasting and $1 \mathrm{~h}$ metabolites to the association of maternal BMI with newborn outcomes. Exp., explained; Metabs, metabolites

as a predictor of the maternal metabolome and relate the maternal metabolome associated with BMI to newborn outcomes. One limitation is that comparing our findings to nonpregnant populations may be subject to confounding, variability in data collection and other systematic study differences that cannot be addressed analytically with the available data. Also, the observational nature of HAPO complicates causal interpretation of metabolites underlying associations between maternal phenotypes and newborn outcomes.

In conclusion, maternal BMI and insulin sensitivity impact a broad array of metabolites and have shared and independent associations with the maternal metabolome. Unlike insulin sensitivity, maternal BMI is independently associated with newborn size at birth. Metabolites associated with maternal BMI independent of maternal glucose include a broad array of metabolites, including lipids and lipid-related metabolites. Some of these metabolites also predicted newborn outcomes in random forest analyses. Future studies examining the impact of maternal obesity on newborn outcomes may require more in-depth analysis of maternal lipid metabolism.

Acknowledgements The authors thank R. Balasubramanian at University of Massachusetts Amherst, School of Public Health and Health Sciences, Department of Biostatistics for helpful discussions on network analyses. 
Data availability Data will be made available by the authors upon request.

Funding This study was funded by grants R01DK095963 from the National Institute of Diabetes and Digestive and Kidney Diseases, and R01-HD34242 and R01-HD34243 from the National Institute of Child Health and Human Development.

Duality of interest BEM has received payment for consultancy to Johnson and Johnson. The authors declare no other duality of interest associated with this manuscript.

Contribution statement VS contributed to study concept and design and interpretation of data. ACR and MN contributed to analysis and interpretation of data. JRB, MJM, RDS, OI, LPL, BEM and CBN contributed to acquisition and interpretation of data. DMS and WLL made substantial contributions to all project components, including study concept and design, acquisition of data, analysis and interpretation of data. All authors made critical intellectual contributions to drafting and/or revising the manuscript and all approved the final version. DMS and WLL are the guarantors of this work and, as such, had full access to all the data in the study and take responsibility for the integrity of the data and the accuracy of the data analysis.

\section{References}

1. HAPO Study Cooperative Research Group (2010) Hyperglycaemia and Adverse Pregnancy Outcome (HAPO) study: associations with maternal body mass index. BJOG 117:575-584

2. Desert R, Canlet C, Costet N, Cordier S, Baonvallot N (2015) Impact of maternal obesity on the metabolic profiles of pregnant women and their offspring at birth. Metabolomics 11:1896-1907

3. Flegal KM, Kruszon-Moran D, Carroll MD, Fryar CD, Ogden CL (2016) Trends in obesity among adults in the United States, 2005 to 2014. JAMA 315:2284-2291

4. Catalano PM, McIntyre HD, Cruickshank JK et al (2012) The hyperglycemia and adverse pregnancy outcome study: associations of GDM and obesity with pregnancy outcomes. Diabetes Care 35: 780-786

5. Cnattingius S, Reilly M, Pawitan Y, Lichtenstein P (2004) Maternal and fetal genetic factors account for most of familial aggregation of preeclampsia: a population-based Swedish cohort study. Am J Med Genet A 130:365-371

6. Nelson SM, Matthews P, Poston L (2010) Maternal metabolism and obesity: modifiable determinants of pregnancy outcome. Hum Reprod Update 16:255-275

7. Schellong K, Schulz S, Harder T, Plagemann A (2012) Birthweight and long-term overweight risk: systematic review and a metaanalysis including 643,902 persons from 66 studies and 26 countries globally. PLoS One 7, e47776

8. Black MH, Sacks DA, Xiang AH, Lawrence JM (2013) The relative contribution of prepregnancy overweight and obesity, gestational weight gain, and IADPSG-defined gestational diabetes mellitus to fetal overgrowth. Diabetes Care 36:56-62

9. Stuebe AM, Landon MB, Lai Y et al (2012) Maternal BMI, glucose tolerance, and adverse pregnancy outcomes. Am J Obstet Gynecol 207:62.e1-62.e7

10. Tyrrell J, Richmond RC, Palmer TM et al (2016) Genetic evidence for causal relationships between maternal obesity-related traits and birthweight. JAMA 315:1129-1140
11. Metzger BE, Lowe LP, Dyer AR et al (2008) Hyperglycemia and adverse pregnancy outcomes. N Engl J Med 358:1991-2002

12. Hayes MG, Urbanek M, Hivert MF et al (2013) Identification of $\mathrm{HKDC} 1$ and BACE2 as genes influencing glycemic traits during pregnancy through genome-wide association studies. Diabetes 62: 3282-3291

13. Scholtens DM, Bain JR, Reisetter AC et al (2016) Metabolic networks and metabolites underlie associations between maternal glucose during pregnancy and newborn size at birth. Diabetes 65 : 2039-2050

14. Urbanek M, Hayes MG, Armstrong LL et al (2013) The chromosome $3 \mathrm{q} 25$ genomic region is associated with measures of adiposity in newborns in a multi-ethnic genome-wide association study. Hum Mol Genet 22:3583-3596

15. HAPO Study Cooperative Research Group (2002) The Hyperglycemia and Adverse Pregnancy Outcome (HAPO) study. Int J Gynaecol Obstet 78:69-77

16. Radaelli T, Farrell KA, Huston-Presley L et al (2010) Estimates of insulin sensitivity using glucose and C-peptide from the hyperglycemia and adverse pregnancy outcome glucose tolerance test. Diabetes Care 33:490-494

17. Scholtens DM, Muehlbauer MJ, Daya NR et al (2014) Metabolomics reveals broad-scale metabolic perturbations in hyperglycemic mothers during pregnancy. Diabetes Care 37:158-166

18. Kind T, Wohlgemuth G, Lee do Y (2009) FiehnLib: mass spectral and retention index libraries for metabolomics based on quadrupole and time-of-flight gas chromatography/mass spectrometry. Anal Chem 81:10,038-10,048

19. Halket JM, Przyborowska A, Stein SE, Mallard WG, Down S, Chalmers RA (1999) Deconvolution gas chromatography/mass spectrometry of urinary organic acids - potential for pattern recognition and automated identification of metabolic disorders. Rapid Commun Mass Spectrom 13:279-284

20. Nodzenski M, Muehlbauer MJ, Bain JR, Reisetter AC, Lowe WL Jr, Scholtens DM (2014) Metabomxtr: an R package for mixturemodel analysis of non-targeted metabolomics data. Bioinformatics 30:3287-3288

21. Benjamini Y, Hochberg Y (2000) On the adaptive control of the false discovery rate in multiple testing with independent statistics. J Educ Behav Stat 25:60-83

22. Kanehisa M, Sato Y, Kawashima M, Furumichi M, Tanabe M (2016) KEGG as a reference resource for gene and protein annotation. Nucleic Acids Res 44:D457-D462

23. Goeman JJ, van de Geer SA, de Kort F, van Houwelingen HC (2004) A global test for groups of genes: testing association with a clinical outcome. Bioinformatics 20:93-99

24. Beisser D, Klau GW, Dandekar T, Muller T, Dittrich MT (2010) BioNet: an R-package for the functional analysis of biological networks. Bioinformatics 26:1129-1130

25. Dittrich MT, Klau GW, Rosenwald A, Dandekar T, Muller T (2008) Identifying functional modules in protein-protein interaction networks: an integrated exact approach. Bioinformatics 24:i223-i231

26. Csardi G, Nepusz T (2006) The igraph software package for complex network research. Interjournal - Complex Systems: 1695

27. Reichardt J, Bornholdt S (2006) Statistical mechanics of community detection. Phys Rev E 74:016110

28. Breiman L (2001) Random forests. Mach Learn 45:5-32

29. Strobl C, Boulesteix AL, Kneib T, Augustin T, Zeileis A (2008) Conditional variable importance for random forests. BMC Bioinforma 9:307

30. Newgard CB, An J, Bain JR et al (2009) A branched-chain amino acid-related metabolic signature that differentiates obese and lean humans and contributes to insulin resistance. Cell Metab 9:311-326

31. Schooneman MG, Vaz FM, Houten SM, Soeters MR (2013) Acylcarnitines: reflecting or inflicting insulin resistance? Diabetes $62: 1-8$ 
32. Huynh J, Xiong G, Bentley-Lewis R (2014) A systematic review of metabolite profiling in gestational diabetes mellitus. Diabetologia 57:2453-2464

33. Dudzik D, Zorawski M, Skotnicki M et al (2014) Metabolic fingerprint of gestational diabetes mellitus. J Proteomics 103:57-71

34. Lowe WL Jr, Karban J (2014) Genetics, genomics and metabolomics: new insights into maternal metabolism during pregnancy. Diabet Med 31:254-262

35. Hajduk J, Klupczynska A, Derezinski P et al (2015) A combined metabolomic and proteomic analysis of gestational diabetes mellitus. Int J Mol Sci 16:30,034-30,045

36. Lindsay KL, Hellmuth C, Uhl O et al (2015) Longitudinal metabolomic profiling of amino acids and lipids across healthy pregnancy. PLoS One 10, e0145794

37. Luan H, Meng N, Liu P et al (2014) Pregnancy-induced metabolic phenotype variations in maternal plasma. J Proteome Res 13:15271536

38. Pinto J, Barros AS, Domingues MR et al (2015) Following healthy pregnancy by NMR metabolomics of plasma and correlation to urine. J Proteome Res 14:1263-1274

39. Kim JY, Park JY, Kim OY et al (2010) Metabolic profiling of plasma in overweight/obese and lean men using ultra performance liquid chromatography and Q-TOF mass spectrometry (UPLC-QTOF MS). J Proteome Res 9:4368-4375

40. Valcarcel B, Ebbels TM, Kangas AJ et al (2014) Genome metabolome integrated network analysis to uncover connections between genetic variants and complex traits: an application to obesity. J R Soc Interface 11:20130908
41. Cheng S, Rhee EP, Larson MG et al (2012) Metabolite profiling identifies pathways associated with metabolic risk in humans. Circulation 125:2222-2231

42. Ho JE, Larson MG, Ghorbani A et al (2016) Metabolomic profiles of body mass index in the Framingham Heart Study reveal distinct cardiometabolic phenotypes. PLoS One 11, e0148361

43. Vidakovic AJ, Jaddoe VW, Gishti O et al (2015) Body mass index, gestational weight gain and fatty acid concentrations during pregnancy: the Generation R Study. Eur J Epidemiol 30:1175-1185

44. Haggarty P (2010) Fatty acid supply to the human fetus. Annu Rev Nutr 30:237-255

45. Tai ES, Tan ML, Stevens RD et al (2010) Insulin resistance is associated with a metabolic profile of altered protein metabolism in Chinese and Asian-Indian men. Diabetologia 53:757-767

46. Catalano PM, Hauguel-De Mouzon S (2011) Is it time to revisit the Pedersen hypothesis in the face of the obesity epidemic? Am J Obstet Gynecol 204:479-487

47. Jang C, Oh SF, Wada S et al (2016) A branched-chain amino acid metabolite drives vascular fatty acid transport and causes insulin resistance. Nat Med 22:421-426

48. Tremblay F, Krebs M, Dombrowski L et al (2005) Overactivation of S6 kinase 1 as a cause of human insulin resistance during increased amino acid availability. Diabetes 54:2674-2684

49. Herrera E, Amusquivar E, Lopez-Soldado I, Ortega H (2006) Maternal lipid metabolism and placental lipid transfer. Horm Res 65(Suppl 3):59-64 